\title{
Implications of Pharmacogenomics in the Current and Future Treatment of Asthma
}

\author{
Thomas J. Morrow, MD
}

\begin{abstract}
BACKGROUND: For more than a generation, managed care has attempted to eliminate variation in care delivery in the hope of producing predictable outcomes. But the population-based, guideline-driven approach may not have fully appreciated the importance of individual behavior (adherence) and environment, as well as individual genetic makeup. Genetic variation in response to currently recommended therapies may require tailoring medication regimens to the individual patient to achieve optimal outcomes.
\end{abstract}

OBJECTIVE: To review the pharmacogenomics of asthma and how they impact the medications utilized for its treatment.

METHODS: A search of PubMed that included the time period from January 1991 through September 2005 and the key terms: asthma pharmacogenetics, asthma genetics, asthma response variability, asthma glucocorticoid resistance, asthma steroid-unresponsive, asthma control, beta-agonist genomics, beta 2-receptor abnormalities, asthma genotypes, and leukotriene inhibitor polymorphisms produced 105 articles. Forty-five were rejected for this subject review by failing the following criteria: (1) results in humans, not animals, (2) provide information about clinical implications as well as description of molecular and cellular mechanism of action or the site of action on the gene, and (3) preference for manuscripts that quantified information/ results over those that just stated that there were observed differences. The remaining 60 references were reviewed, and 7 references were added after peer review.

RESULTS: There are now limited examples of gene polymorphisms that can influence responses to beta 2-agonists, glucocorticosteroids, and leukotriene modifiers in patients with asthma. Gene mutations that are known to alter the response to asthma therapy include Arg/Arg atr position 16, mutations of LTC4S, ALOX5, and GR/NR3C1, increased expression of GR , CRHR1 variants, and mutations in CYP1A2 (-22964 [G/A]), and T 314 allele for histamine $\mathrm{N}$-methyltransferase. Some of the effects associated with these mutations are increased/decreased response to therapy, glucocorticoid resistance, decreased theophylline clearance and possible toxicity, and increased bronchoconstriction.

CONCLUSIONS: Understanding the impact of genetic variations on response to therapy may ultimately improve treatment outcomes for patients with asthma. However, despite substantial progress, no individual gene polymorphisms have been associated with altered responses to asthma treatment in large numbers of patients. It is not yet possible to tailor medication therapy for asthma based on genetic characteristics of individual patients.

KEYWORDS: Asthma, Genetics, Polymorphisms, Treatment response, Outcomes

J Manag Care Pharm. 2007;13(6):497-505

Copyright@ 2007, Academy of Managed Care Pharmacy. All rights reserved.

\section{What is already known ahout this subject}

- There is substantial interpatient variation in responses to different asthma therapies.

- Genetic mutations associated with altered responses to commonly used asthma therapies have been identified.

What this study adds

- Although genetic variants have been recognized that affect asthma treatment response, pharmacogenomic evaluation is still too immature to implement population-wide, individualized asthma therapy.

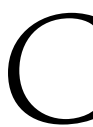
linical practice has evolved from anecdotal case reports, to collections of signs and symptoms, to evidencebased medicine. This approach has generally embraced population-based approaches to care to produce a more consistent outcome from different providers. Treatment of both acute and chronic disease is now driven by guidelines based on results from large-scale, well-controlled clinical trials. Examples of well-known guidelines include those for treatment of hypertension, dyslipidemia, diabetes, and depression..$^{1-4}$

In general, treatment guidelines centralize information about phenotypic characteristics (e.g., sex, age, and body weight), patient and family history, and disease severity (e.g., blood pressure and cholesterol level) to drive treatment decisions in a standardized manner. ${ }^{1,2}$ This has led to a dramatic improvement in overall care as it has diminished the variability of individual practitioners in their application of evidence.

The sequencing of the human genome was a fundamentally important step in the evolution of medicine and a quantum leap in our understanding of genes, their association with specific diseases, and new targets of pharmacotherapy. Since the completion of the draft of the human genome in early 2001, genebased therapies have begun to influence patient care. Advances in the management of hepatitis $C,{ }^{5}$ schizophrenia, ${ }^{6}$ leukemia, ${ }^{7}$ prostate cancer, ${ }^{8}$ lung cancer, ${ }^{9}$ and breast cancer $^{10}$ have all resulted from increased understanding of the genes associated with these diseases. Understanding the manner in which a given patient's genetic inheritance may influence response to therapy has increased attention on individualization of therapy based on such information. ${ }^{10}$

The highly complex respiratory system is an important pathway for the entry of disease-causing vectors, including viruses, bacteria, fungi, toxins, and antigens. ${ }^{11}$ Interpatient 
variability in response to treatments for respiratory diseases, such as asthma, is very high, ${ }^{12,13}$ but efforts to understand potential genetic sources of this variability have lagged behind those for other conditions. The objective of this review is to highlight the pharmacogenomics of asthma and how they impact the medications utilized to treat this disease.

\section{Overview of Asthma}

\section{Pathophysiology}

Asthma is a chronic inflammatory disease of the airways that is characterized by intermittent and at least partially reversible bronchoconstriction, as well as by airway hyperresponsiveness to a wide variety of stimuli. ${ }^{13}$ The inflammatory features characteristic of asthma include infiltration of the airways by inflammatory cells that release various cytokines and inflammatory mediators. These mediators result in an increase in airway edema and mucus secretion, hypertrophy and hyperplasia of airway smooth muscle, and increased airway vascularity, all of which contribute to airflow obstruction. ${ }^{13,14}$ There is wide variability in the pathophysiologic features apparent in different patients with asthma. In many patients, eosinophils are the predominant inflammatory cell type, while in others, neutrophils rather than eosinophils have been shown to be present as the dominant inflammatory cell. ${ }^{15}$ Variability in response to medications is also commonly seen in asthmatics. ${ }^{3}$ There are many potential reasons for this variability. As noted above, asthma is typically characterized by eosinophil activation and infiltration of the airways, but some patients have increased neutrophils and lack eosinophils in their airways. ${ }^{13}$ Such patients may have decreased responses to leukotriene response modifiers and/or inhaled corticosteroids.

\section{Epidemiology}

Asthma is a very common disease associated with high morbidity. Review of worldwide data indicates that the prevalence of asthma has increased substantially over the last 20 years, but the reasons for this are not clear. ${ }^{16}$ Results from the United States indicate that the prevalence of asthma had increased by $75 \%$ from 1980 to $1994^{17}$ and asthma now affects $8 \%$ to $10 \%$ of the U.S. population..$^{18}$ In a survey of more than 42,000 U.S. households, $30 \%$ of patients with mild to moderate disease and $70 \%$ of those with moderate to severe disease, based on symptoms, reported some level of functional impairment. ${ }^{19}$ In 1998, the direct and indirect costs associated with medical care of patients with asthma exceeded $\$ 11$ billion ( $\$ 7.5$ billion and $\$ 3.8$ billion, respectively). ${ }^{20}$

\section{Asthma Diagnosis, Therapy, and Current Treatment Guidelines Diagnosis}

Accurate diagnosis is the critical component in the management of asthma. Generally, asthma presents episodic symptoms of airflow obstruction that are at least partially reversible and not attributable to other pathologies. Chronic obstructive pulmonary disease, vocal cord dysfunction in adults, and cystic fibrosis and aspiration in children, must be ruled out in the differential diagnosis of asthma. Spirometric studies utilizing prebronchodilator and postbronchodilator therapy measuring forced expiratory volume in 1 second $\left(\mathrm{FEV}_{1}\right)$ and peak flow are valuable in measuring reversibility and classifying disease severity. ${ }^{21}$ Allergens and irritants that can trigger symptoms or exacerbations should be identified and removed or exposure limited..$^{21}$ Thus, while certain features are considered characteristic of asthma, heterogeneity exists in terms of pathologic presentation and response to therapy. It seems logical that genetic variability may explain some of this heterogeneity.

\section{Pharmacotherapy}

The goal of pharmacotherapy is to successfully maintain normal activity levels, including exercise; control chronic and nocturnal symptoms; optimize pulmonary function; prevent acute episodes of asthma; and avoid adverse effects of asthma medications. Medications used to treat asthma can be divided into 2 general groups: acute-relief medications (i.e., short-acting beta-agonists, and systemic glucocorticosteroids) and chronic-use medications (inhaled corticosteroids, cromolyn/nedocromil, leukotriene modifiers, long-acting beta-agonists, methylxanthines, and omalizumab). ${ }^{21-24}$

\section{Current Treatment Guidelines and Treatment Efficacy}

The National Asthma Education and Prevention Program guidelines recommend a stepwise approach to pharmacologic therapy, whereby the amount and frequency of medications are dictated by the severity of the asthma and directed toward suppression of increasing airway inflammation. According to these guidelines, therapy is initiated aggressively to establish prompt control and then slowly stepped-down to minimize the risk of adverse events without sacrificing efficacy. ${ }^{21}$

Achievement of treatment goals are less than optimal in many patients. ${ }^{25,26}$ For some this may be due to poor adherence to treatment guidelines, but for a small subgroup, this may be due, in part, to genetic polymorphisms as well as the fact that disease severity may be misclassified in some patients with asthma, resulting in inappropriate therapy. ${ }^{27,28}$ Even treatment that is fully consistent with current guidelines fails to control asthma in some patients. Results of a randomized, stratified, double-blind, parallel-group study of 3,421 patients with uncontrolled asthma showed that fully optimized, long-term drug therapy with inhaled corticosteroids or inhaled corticosteroids plus a longacting beta-agonist controlled approximately $75 \%$ of this group. While these results suggest that the majority of patients could reach guideline-defined measures of control, approximately $25 \%$ of these managed patients could not achieve control as defined by the Global Initiative for Asthma and the National Institutes of Health. ${ }^{29}$ These results suggest that other factors, 
such as severity of disease, concurrent illness, environmental exposures, medication noncompliance, and interpatient genetic variability in response to asthma therapy may play important roles in treatment efficacy. It is reasonable to suggest that current treatment guidelines for asthma therapy should be reviewed in light of the latest information about genetic determinants of responsivity to commonly used asthma therapies.

Genetic Determinants of Responsitivity to Asthma Therapy Genetic factors, including polymorphism in a gene or a random DNA position (single nucleotide polymorphism [SNP]), or in a series of associated alleles, play a role in determining heterogeneous responses to pharmacological treatment among patients with asthma. Drug therapy tailored to an asthmatic patient's genotype may result in a clinically important increase in efficacy and a reduction in adverse events. ${ }^{30}$

\section{Specific Genetic Mutations That Alter Responses to Different Asthma Therapies}

Gene mutations that alter responses to asthma therapy are summarized in Table 1 and described in detail in the following sections.

\section{Beta 2-Agonists}

Beta 2-agonists are important bronchodilator drugs commonly used in the treatment of asthma. The beta 2-adrenoreceptor gene is expressed in bronchial smooth muscle cells and induces dilation in response to endogenous catecholamine or exogenous triggers. Several polymorphisms have been described in this gene, which is located on the chromosome 5q31-32. Three coding polymorphisms, located at positions 16,27 , and 164 , have been studied. ${ }^{30}$

Clinical studies have indicated that the Arg/Arg genotype for residue 16 of the beta 2-receptor alters responses to treatment and disease severity in patients with asthma. Results from one study showed that albuterol-evoked $\mathrm{FEV}_{1}$ was higher and the response was more rapid in Arg16 homozygotes compared with carriers of the Gly16 variant (18\% increase versus $4.9 \%$ increase, $P<0.03) .{ }^{31}$ Similarly, spirometric assessment of 269 participants in a longitudinal study of asthma indicated that homozygotes for Arg16 were 5.3 times more likely than Gly16 homozygotes to respond $\left(>15.3 \%\right.$ increase in $\left.\mathrm{FEV}_{1}\right)$ to challenge with $180 \mathrm{mcg}$ albuterol. $^{32}$

In contrast, clinical trial results have indicated a decreased response to longer-term beta 2-agonist treatment among patients with Arg/Arg genotype for residue 16 of the beta 2-receptor as well as increased risk of exacerbations among patients with this genotype who were treated with a short-acting beta 2-agonist. 33,34 The Beta-Adrenergic Response by Genotype trial was designed to establish a genotype-dependent effect of albuterol use on airway function. Patients with mild asthma were enrolled based on clinical criteria and their genotype (Arg/Arg or homozygous for glycine [Gly/Gly]) at the locus encoding the 16th amino acid in the beta 2-adrenocepter. ${ }^{33}$ Results showed that patients with the Arg/Arg genotype had increased peak expiratory flow rates (PEFR) when beta 2-agonists were withdrawn as a rescue inhaler and replaced with ipratropium bromide. In contrast, patients with the Gly/Gly genotype showed good responses to beta 2-agonist therapy, that reversed when it was withdrawn. During randomized treatment, patients with the Gly/Gly genotype

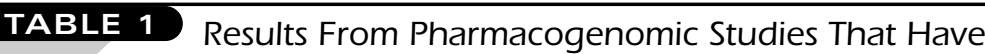 Provided Information Relevant to the Treatment of Asthma}

\begin{tabular}{|c|c|c|}
\hline Drug Class & Mutation & Effect \\
\hline Beta 2-agonists & Arg/Arg at position 16 & $\begin{array}{l}\text { - Increased acute response to albuterol } 1^{31,32} \\
\text { - Increased risk for exacerbations }{ }^{33,34} \\
\text { - Decreased response to long-term treatment with short-acting beta-agonists }{ }^{28} \\
\text { - Increased PEFR when short-acting beta-agonist withdrawn }{ }^{33,35}\end{array}$ \\
\hline \multirow[t]{2}{*}{ Leukotriene response modifier } & LTC4S mutation & $\begin{array}{l}\text { - Decreased response to zafirlukast }{ }^{36} \\
\text { - Decrease in } \mathrm{FEV}_{1} \text { with zafirlukast }{ }^{36}\end{array}$ \\
\hline & ALOX5 mutation & - Decreased response to zafirlukast ${ }^{36}$ \\
\hline \multirow[t]{3}{*}{ Glucocorticoids } & GR/NR3Cl mutations & - Glucocorticoid resistance ${ }^{37}$ \\
\hline & Increased expression of GR & $\begin{array}{l}\text { - Decreased ability of inhaled corticosteroids to decrease expression of } \\
\text { inflammatory cytokines by pulmonary cells } s^{38-40}\end{array}$ \\
\hline & CRHRl variants & - Increased response to inhaled corticosteroids ${ }^{41}$ \\
\hline \multirow[t]{2}{*}{ Theophylline } & CYP1A2 (-2964 [G/A]) & - Decreased clearance and possible toxicity ${ }^{42}$ \\
\hline & $\begin{array}{l}\text { T } 314 \text { allele for histamine N-methyl- } \\
\text { transferase }\end{array}$ & - Increased bronchoconstriction ${ }^{30}$ \\
\hline
\end{tabular}


had an increase in morning PEFR of $14 \mathrm{~L} /$ min versus placebo with regularly scheduled albuterol. Patients with the Arg/Arg genotype had lower morning PEFR (-10 L/min) during treatment with albuterol than during the placebo period, when albuterol use was limited. The genotype-attributable treatment difference was thus $-24 \mathrm{~L} / \mathrm{min} .{ }^{33}$ This information indicates that chronic treatment with a short-acting beta 2-agonist should probably be avoided in asthma patients with the Arg/Arg genotype. ${ }^{33}$ It is estimated that $15 \%$ ( $16 \%$ of whites and $20 \%$ of blacks) of the population is homozygous for Arg16. ${ }^{35,43}$

A retrospective analysis of relationships between polymorphisms at codons 16 and 27 of the beta 2-adrenoceptor and clinical outcomes in a randomized, placebo-controlled, crossover trial of regularly scheduled salbutamol and salmeterol in 115 patients with mild to moderate asthma indicated that patients with the Arg/Arg genotype had more frequent exacerbations during salbutamol treatment than with placebo (1.91 versus $0.81, P=0.005) \cdot{ }^{34}$ No significant treatment-related differences occurred for heterozygous Arg-Gly patients or homozygous Gly-16 patients. ${ }^{34}$

\section{Leukotriene Response Modifiers}

Leukotrienes are released from mast cells, eosinophils, and other inflammatory cells in the airways of patients with asthma. ${ }^{44}$ Cysteinyl leukotrienes, $\mathrm{C}_{4}, \mathrm{D}_{4}$, and $\mathrm{E}_{4}$, released primarily from activated eosinophils and mast cells, are potent contributors to the physiological and pathological changes characteristic of asthma. ${ }^{45}$ They are several orders of magnitude more potent than acetylcholine and histamine as contractile agonists of human airways. ${ }^{45,46}$ Leukotrienes increase microvascular permeability, modulate the primary afferent nerve fibers, stimulate mucus release, slow mucus transport, and decrease the activity of human respiratory cilia. ${ }^{45}$

Antileukotriene therapies inhibit synthesis of leukotrienes through 5-lipoxygenase (ALOX5) inhibition or by blocking the cysteinyl leukotriene receptor. ${ }^{46}$ The -444 leukotriene C4 synthase gene polymorphism (LTC4S) has been correlated with the response of asthma patients to zafirlukast, a leukotriene receptor antagonist. ${ }^{36}$ Anderson and colleagues genotyped asthma patients for polymorphisms in the promoter region of ALOX5 and LTC4S. These individuals were participating in a randomized, double-blind, parallel study of inhaled fluticasone (88 mcg twice daily) and zafirlukast (20 mg twice daily). Results showed that subject's homozygous for mutations in either ALOX5 or LTC4S had a reduced response to zafirlukast therapy. Results from this same study of 68 patients with mild asthma showed that zafirlukast had no activity in LTC4S C/C homozygotes compared with heterozygotes and carriers of the A allele based on percentage change in $\mathrm{FEV}_{1}(-3 \%,+9 \%,+9 \%$, respectively). ${ }^{36} \mathrm{~A}$ third study of 23 patients with chronic, severe asthma indicated a different relationship between the LTC4 genotype and response to zafirlukast. In this trial, the $\mathrm{FEV}_{1}$ response to zafirlukast was increased in heterozygotes and C/C homozygotes while A/A homozygotes had a decrease in FEV with zafirlukast. ${ }^{47}$ The reasons for the different patterns of results are not clear, although it may be attributed to differences between population types. Among 114 individuals receiving high-dose zafirlukast, 104 wild-type or heterozygous patients had an $18.8 \%$ improvement in $\mathrm{FEV}_{1}$ after 1 week of treatment. In contrast, 10 patients with the mutant genotype had no benefit from active treatment, as measured by an average change in $\mathrm{FEV}_{1}$ of $-1.2 \%$. None of the patients with the mutant genotype at the ALOX 5 core promoter locus manifested a $>12 \%$ improvement in $\mathrm{FEV}_{1}$ at the end of the treatment period. ${ }^{48}$

\section{Glucocorticoids}

Glucocorticoids are the most potent anti-inflammatory drugs used for asthma treatment. ${ }^{30}$ They act by binding to an intracellular glucocorticoid receptor (GR) to form a complex. The receptor-ligand complex translocates to the nucleus where it regulates gene expression, decreasing transcription of various proinflammatory proteins and increasing transcription of anti-inflammatory proteins. ${ }^{30,40}$ Glucocorticoids also increase transcription of beta 2-adrenoceptors and muscarinic receptors. This increase in transcription may help to shift airway regulation from vagally mediated bronchoconstriction to sympathetically mediated bronchorelaxation. ${ }^{30}$

The clinical efficacy of glucocorticoid therapy is derived from a combination of anti-inflammatory effects in the lung, reduction of inflammatory cell survival, and inhibition of inflammatory cytokine production. ${ }^{49}$ Despite their well-known efficacy, there is a subset of asthmatic patients who are unresponsive to corticosteroids. ${ }^{49-52}$ These patients demonstrate persistent respiratory symptoms, nocturnal exacerbations, persistent airway obstruction, and inflammation, even though their treatment includes high doses of systemic glucocorticoids. ${ }^{49-52}$ Clinical studies have shown about 5\% to $10 \%$ of all patients with asthma and up to $35 \%$ of those with severe disease have reduced responses to glucocorticosteroid therapy. ${ }^{50-52}$ African Americans may appear to have a racial predisposition to decreased responsitivity to glucocorticosteroid therapy, which was approximately $40 \%$ in poorly controlled patients. ${ }^{52}$

It has been shown that some glucocorticoid-resistant patients have abnormalities in the activity of proinflammatory transcription factors AP-1 and NF-KB. ${ }^{30}$ Both AP-1 and NF$\mathrm{KB}$ act by inducing the transcription of chemoattractants, cytokines, cytokine receptors, and cell adhesion molecules. ${ }^{40}$ Many cases of glucocorticoid resistance may be due to mutations or polymorphisms present in the glucocorticoid receptor gene (GR/NR3C1) ${ }^{37}$ A total of 15 missense, 3 nonsense, 3 frameshift, 1 splice site, and 2 alternative spliced mutations have been reported in the $\mathrm{NR} 3 \mathrm{Cl}$ gene. These mutations have been associated with glucocorticoid resistance. ${ }^{37}$

There are 2 naturally occurring isoforms of the NR3Cl: GR $\alpha$ 
(functional) and GR $\beta$ (no hormone-binding ability). ${ }^{37}$ The glucocorticoid-GR $\alpha$ complex can directly or indirectly alter gene transcription by binding to specific DNA sites or through transcription factor activation. GR $\alpha$ may also be involved with down-regulation of proinflammatory mediators and upregulation of anti-inflammatory mediators. GR $\beta$ is thought to act as an endogenous inhibitor of glucocorticoid action. ${ }^{37,38,40}$ Leung and colleagues ${ }^{38}$ carried out bronchoalveolar lavage (BAL) in 6 steroid-resistant and 6 steroid-sensitive patients with asthma before and after 1 week of treatment with $40 \mathrm{mg} /$ day prednisone. Before prednisone therapy, there were significantly greater numbers of BAL cells expressing IL (interleukin)-2 mRNA $(P<0.01)$ and IL-4 mRNA $(P<0.05)$ in steroid-resistant patients with asthma, as compared with steroid-sensitive patients..$^{38}$ There were no between-group differences observed in the numbers of BAL cells expressing interferon (IFN)- $\gamma$ or IL-5 mRNA expression. After 1 week of prednisone treatment, IL-2 expression was not significantly altered in either group. However, steroid-sensitive patients had a significant decrease in the numbers of BAL cells expressing mRNA for IL-4 $(P<0.01)$ and IL-5 $(P<0.001)$, and a rise in the numbers of IFN- $\gamma$ mRNA + cells $(P<0.01)$. In contrast, after prednisone treatment, the patients with steroid resistance had no significant change in either the number of BAL cells expressing mRNA for IL-4 or IL-5. ${ }^{38}$

An imbalance in the activity of either isoform due to a genetic anomaly may increase the risk of glucocorticoid resistance. The synthesis of glucocorticoid receptors is strongly influenced by interleukins. Genetic polymorphisms that alter expression of several interleukins have been associated with reduced responsivity to corticosteroids in patients with asthma. ${ }^{38,40}$

To date, 2 types of steroid-resistant (SR) asthma have been identified: type I ( $>95 \%$ of cases) is cytokine induced and is associated with increased expression of GR $\beta$, a less active GR isoform, and type II ( $<5 \%$ of cases) is due to low numbers of GRs. Clinically, Type I SR asthmatic patients present with severe side effects, including adrenal gland suppression and Cushingoid features. Type II SR asthmatics have a generalized primary cortisol resistance and do not develop steroid-induced side effects. ${ }^{49}$

Other genetic factors may impact response to corticosteroid therapy. CRHRl is the primary receptor mediating the release of adrenocorticotrophic hormone, which regulates endogenous cortisol levels and genetic variation in CRHRl that is associated with improved pulmonary function response to inhaled corticosteroids. ${ }^{41}$ The mean percentage change in $\mathrm{FEV}_{1}$ for those homozygous for the minor allele was $13.3 \%$ versus $5.5 \%$ for those homozygous for the wild-type allele. ${ }^{41}$

\section{Other Therapies}

Genetic factors may also influence the safety and efficacy of other commonly used asthma treatments. Cytochrome p450 (CYP) $1 \mathrm{~A} 2$ is involved in the metabolism of theophylline, and a polymorphism for the gene encoding this enzyme, -2964 (G/A), has been correlated with reduced theophylline clearance versus that in patients with the G/G genotype. Thus, theophylline may require reduced dosing in patients with the A allele at site -2964 (G/A) in the CYP1A2 gene to avoid possible toxicity. ${ }^{42}$

Histamine is a bronchoconstrictor involved in the pathogenesis of asthma, and histamine $\mathrm{N}$-methyltransferase plays a central role in histamine catabolism in bronchial tissue. The T 314 allele of the gene for histamine N-methyltransferase results in decreased enzyme activity and possibly also increased bronchoconstriction in patients with asthma. It may be important to use antihistamines that do not, themselves, inhibit this enzyme in asthma patients with the T 314 allele. ${ }^{30}$

Eotaxin (chemokine, CC motif, ligand; CCL11) is a potent eosinophil chemoattractant that plays a significant role in the pathology of asthma. Recent results have indicated that the genetic variation at the CCL11 locus is an important determinant of serum total IgE levels among patients with asthma, ${ }^{53}$ and it is reasonable to suggest that CCL11 genotype may influence the response to medications that exert their effect via IgE receptors. Omalizumab is a recombinant anti-IgE antibody therapy for asthma targeted at patients with elevated IgE levels. ${ }^{54}$ Thus, in theory, this genotype might predict a positive response to omalizumab in asthma patients, but studies carried out, to date, have not evaluated this possibility.

The results presented in this section indicate that there are numerous genetically influenced pathways that contribute to the wide interpatient variability in response to commonly prescribed asthma therapies. Currently, national guidelines do not suggest testing for these variations. These and other undiscovered variations will undoubtedly play a significant role in the future of defining the proper therapy for each individual. Additional research is required to more accurately predict therapeutic responses based on individual patient genotypes.

Integration of Molecular Diagnostics With Therapeutics Economic Considerations for Pharmacogenomics

Economic considerations need to be considered in the application of pharmacogenomics to clinical therapy. A set of cost-effectiveness criteria has been proposed to determine when pharmacogenomics is appropriate in selection of therapy and can act as a guide for future research. These criteria include: (1) disease has a severe outcome, defined as a significant impact on the quality of life, or has expensive medical care costs, or has a high mortality; (2) a drug's response is currently not monitored or there is difficulty in monitoring the response; (3) there is a strong association between gene variant and clinically relevant outcomes; (4) a rapid and relatively inexpensive assay is available; and (5) variant allele frequency is relatively high (Table 2)..$^{55}$ These criteria could eventually be considered in the drug selection processes utilized by managed health care plans.

The majority of managed health care plans use prior- 


\begin{tabular}{l|l|l}
\hline \multicolumn{1}{c}{ TABLE 2 } & $\begin{array}{l}\text { Framework for Evaluating the Potential Cost-Effectiveness } \\
\text { of Pharmacogenomic-Based Therapies } 55\end{array}$ \\
\hline Factors & Characteristics Favoring Cost-Effectiveness & Current Status in Asthma Disease Condition \\
\hline Severity of outcome avoided & $\begin{array}{l}\text { Severe outcomes, which include high mortality, significant } \\
\text { impact on quality of life, or expensive health care costs }\end{array}$ & $\begin{array}{l}\text { If identify patient as steroid insensitive, then what alternative } \\
\text { can be used to improve quality of life? }\end{array}$ \\
\hline Drug monitoring & $\begin{array}{l}\text { Drug-response monitoring that is currently not practiced or } \\
\text { difficult }\end{array}$ & $\begin{array}{l}\text { Monitoring accomplished by home peak-flow logs and } \\
\text { symptom-based asthma action plans }\end{array}$ \\
\hline Genotype-phenotype association & $\begin{array}{l}\text { Strong association between gene variant and clinically } \\
\text { relevant outcomes }\end{array}$ & How clinically relevant are IL-4 and IL-5 levels? \\
\hline Assay & Availability of a rapid and relatively inexpensive assay & How much are the current assays? Availability? \\
\hline Polymorphism & Relatively high frequency of variant allele & See Table 1 \\
\hline IL=interleukin. &
\end{tabular}

authorization (PA) programs to ensure appropriate utilization of specified drugs and to reinforce prescription guidelines. As genomic testing advances from a research tool to a clinical tool, managed care plans will be tasked to integrate the testing into the PA process. Adding the above criteria will remind physicians to consider the value of genetic testing when expected therapy outcomes are not achieved with optimally applied, comprehensive management regimens using current evidencebased treatment guidelines combined with a well-designed and well-run disease management program. It will also give a level of confidence that targeted therapies are being used in the most appropriate manner.

\section{Current Application of Pharmacogenomics}

Genetic and genomic testing and analysis are already being incorporated into treatment decisions for patients with many diseases. For example, estrogen receptor and progesterone receptor status are used to select breast cancer patients likely to respond to hormone therapy. ${ }^{56}$ Human epidermal growth factor receptor (HER-2 [c-erbB2/neu]) expression is also used to help guide treatment selection in women with breast cancer, with significant overall survival benefit following chemotherapy in targeted patients. ${ }^{57}$ Results from studies of patients with other malignancies demonstrated clear genetic determination of responses to therapy. ${ }^{58,59}$

Genotype has also been demonstrated to influence responses to therapy in hepatitis $C$ patients. Results from a recent study of patients with chronic hepatitis $C$ indicated that increased hepatic expression of suppressor of cytokine signaling (SOCS)-3 mRNA was significantly associated with lack of response to IFN treatment. ${ }^{60}$

Genetic polymorphisms that influence responsiveness to antidepressant therapy have also been identified. Although substantial further research is required, in the future, pharmacogenetic approaches may potentially affect the treatment of major depression. ${ }^{61}$ Specific genetic variants have also been associated with responses to therapy in patients with schizophrenia. ${ }^{62}$
An early yet growing body of evidence shows that incorporating our understanding of genomics into clinical practice can lead to clinical benefit. Genetic predictors of responses to specific therapies could be helpful in patients with asthma and clinicians should be educated regarding these determinants. ${ }^{63,64}$

At present, there has been little integration of genomics and genetic testing for determination of best approaches to therapy for patients with asthma. However, results from several studies might have "set the stage" for this approach. For example, it has been noted that the association of the CRHRl gene, as well as 1 specific haplotype within the CRHRl gene, with the degree of response to inhaled corticosteroids, may provide the basis for a first step in the development of individualized therapy for asthma. ${ }^{65}$

However, the applicability of genomic and genetic testing faces significant challenges. Patients are likely to be uncomfortable without the presence of confidentiality safeguards. Physicians will be faced with a bewildering array of testing from competing vendors. Managed care companies will face difficulties in tracking and managing the utilization of these complicated tests due to potentially high costs and lack of an adequate coding system for billing. They will also face difficulties in coordinating all of the contracts in a rapidly expanding field. Pharmaceutical companies will face situations in which decisions to control utilization of their products are influenced by testing that is likely to be less than $100 \%$ sensitive or $100 \%$ specific. Patients will be caught in the middle.

It is obvious that standards of care will be sorely needed to guide this process. Most importantly, it is essential that future clinical trials demonstrate that the clinical benefits achieved with therapy selected on the basis of pharmacogenetic analysis justify the cost of testing. A recent modeling study carried out by Stallings and colleagues compared the annualized per-patient cost testing all asthma patients for a nonresponse genotype prior to treating versus no testing. They estimated that the savings associated with the testing strategy ranged from $\$ 200$ to $\$ 767$ 
per patient and concluded that testing costs would be more than offset by avoided nonresponse costs. ${ }^{66}$

\section{Conclusions}

We now have more information about the genetic underpinnings of interpatient variability in response to therapies used in patients with asthma. There are clear examples of gene polymorphisms that can influence responses to beta 2-agonists, glucocorticosteroids, and leukotriene modifiers. However, it must be remembered that despite substantial progress, no individual gene polymorphisms have been associated with altered responses to treatment in large numbers of patients, which is critical to obtain prior to fielding gene testing. ${ }^{67}$

Emerging results for a wide range of diseases, including asthma, indicate that standards of care established in treatment guidelines may not be uniformly applicable to the entire population of patients with a given disease because of multiple causes, one of which is gaining recognition: genetic variation in treatment response. In asthma, there is significant genetically determined variation in response to the 3 main modes of therapy: inhaled corticosteroids, beta 2-agonists, and leukotriene response modifiers. These genetically determined variations in response are important to keep in mind when clinicians make modifications to therapeutic regimens for asthma therapy to achieve control of symptoms and exacerbations. Understanding the impact of genetic variations on response to therapy has the potential to improve care, decrease side effects, and improve patient outcomes.

Managed care physicians and patients will soon enter a new era of complexity that will require significant education. It is important that they understand the therapeutic as well as the social and economic implications of our increased understanding of both the genetics of disease and responses to specific therapies. There are important and difficult ethical issues related to genetic testing (e.g., cost insurability, employability, medical prognosis and treatment decisions based on genetic information) that must be addressed by both health care providers and society in general.

\section{Author}

THOMAS J. MORROW, MD, is director, Value-Based Health Care, Genentech, Inc., South San Francisco, California, and is immediate past president of the National Association of Managed Care Physicians.

AUTHOR CORRESPONDENCE: Thomas J. Morrow, MD, Director, Value-Based Health Care, Genentech, Inc., 320 Pilgrimage Point, Alpharetta, GA 30022. Tel: (404) 583-6028; Fax: (770) 663-0754; E-mail: thomasmorrowmd@hotmail.com

\section{DISCLOSURES}

No outside funding supported this research. Author Thomas J. Morrow worked as an independent consultant and had a primary affiliation with Teva Neuroscience during the time that this article was prepared. After submission of this article, the author joined Genentech as a director in the Value-Based Health department.

\section{REFERENCES}

1. Chobanian AV, Bakris GL, Black HR, et al. Seventh Report of the Joint National Committee on Prevention, Detection, Evaluation, and Treatment of High Blood Pressure. Hypertension. 2003;42:1206-52.

2. Executive summary of The Third Report of the National Cholesterol Education Program (NCEP) Expert Panel on Detection, Evaluation, and Treatment of High Blood Cholesterol in Adults (Adult Treatment Panel III). JAMA. 2001;285:2486-97.

3. American Diabetes Association. Standards of medical care in diabetes. Diabetes Care. 2005;28(suppl 1):S4-36

4. American Psychiatric Association. Diagnostic and Statistical Manual of Mental Disorders. Washington, DC: American Psychiatric Association; 2000.

5. Chen L, Borozan I, Feld J, et al. Hepatic gene expression discriminates responders and nonresponders in treatment of chronic hepatitis $C$ viral infection. Gastroenterology. 2005; 128:1437-44

6. Kondo T, Mihara K, Suzuki A, Yasui-Furukori N, Kaneko S. Combination of dopamine D2 receptor gene polymorphisms as a possible predictor of treatment-resistance to dopamine antagonists in schizophrenic patients. Prog Neuropsychopharmacol Biol Psychiatry. 2003;27:921-26.

7. Moshynska O, Sankaran K, Saxena A. Molecular detection of the G(-248)A BAX promoter nucleotide change in B cell chronic lymphocytic leukaemia. Mol Pathol. 2003;56:205-09

8. Buchanan G, Irvine RA, Coetzee GA, Tilley WD. Contribution of the androgen receptor to prostate cancer predisposition and progression. Cancer Metastasis Rev. 2001;20:207-23.

9. Seve P, Dumontet C. Chemoresistance in non-small cell lung cancer. Curr Med Chem Anti-Canc Agents. 2005;5:73-88.

10. Lymberis SC, Parhar PK, Katsoulakis E, Formenti SC. Pharmacogenomics and breast cancer. Pharmacogenomics. 2004;5:31-55.

11. Gerritsen J. Host defence mechanisms of the respiratory system. Paediatr Respir Rev. 2000;1:128-34

12. Sayers I, Hall IP. Pharmacogenetic approaches in the treatment of asthma. Curr Allergy Asthma Rep. 2005;5:101-08.

13. Peters SP. Heterogeneity in the pathology and treatment of asthma. Am J Med. 2003;115(suppl 3A):49S-54S.

14. Houtman R, van den Worm E. Asthma, the ugly duckling of lung disease proteomics? J Chromatogr B Analyt Technol Biomed Life Sci. 2005;815:285-94.

15. ten Brinke A, Zwinderman AH, Sterk PJ, Rabe KF, Bel EH. "Refractory" eosinophilic airway inflammation in severe asthma: effect of parenteral corticosteroids. Am J Respir Crit Care Med. 2004;170:601-05.

16. Keller MB, Lowenstein SR. Epidemiology of asthma. Semin Respir Crit Care Med. 2002;23:317-29.

17. Castro HJ, Malka-Rais J, Bellanti JA. Current epidemiology of asthma: emerging patterns of asthma. Allergy Asthma Proc. 2005;26:79-82.

18. Busse W, Banks-Schlegel S, Noel P, Ortega H, Taggart V, Elias J. Future research directions in asthma: an NHLBI Working Group report. Am J Respir Crit Care Med. 2004;170:683-90.

19. Fuhlbrigge AL, Adams RJ, Guilbert TW, et al. The burden of asthma in the United States: level and distribution are dependent on interpretation of the national asthma education and prevention program guidelines. Am J Respir Crit Care Med. 2002;166:1044-49.

20. National Institutes of Health. Data Fact Sheet: Asthma Statistics. Bethesda, MD: National Heart, Lung, and Blood Institute; 1999. 
21. National Institutes of Health. National Asthma Education and Prevention Program. Expert Panel Report 2: Guidelines for the Diagnosis and Management of Asthma. Bethesda, MD: National Heart, Lung, and Blood Institute; 1997. Report no.: 97-4051.

22. American Hospital Formulary Service. McEvoy GK, Snow EK, Kester L, eds. AHFS Drug Information. Bethesda, MD: American Society of Health-System Pharmacists; 2006:2718-89.

23. National Institutes of Health. National Asthma Education and Prevention Program. Expert Panel Report: Guidelines for the Diagnosis and Management of Asthma-Update on Selected Topics, 2002. Bethesda, MD: National Heart, Lung and Blood Institute; 2002. Report no.: 02-5075.

24. Global Strategy for Asthma Management and Prevention 2006. Global Initiative for Asthma (GINA) Web site. Available at: www.ginasthma.org. Accessed February 26, 2007.

25. Gendo K, Lodewick MJ. Asthma economics: focusing on therapies that improve costly outcomes. Curr Opin Pulm Med. 2005;11:43-50.

26. Stempel DA, Roberts CS, Stanford RH. Treatment patterns in the months prior to and after asthma-related emergency department visit. Chest. 2004:126:75-80.

27. Holgate ST, Price D, Valovirta E. Asthma out of control? A structured review of recent patient surveys. BMC Pulm Med. 2006;6 (supp 1):S2.

28. Telleria JJ, Blanco-Quiros A, Muntion S, et al. Tachyphylaxis to beta2-agonists in Spanish asthmatic patients could be modulated by beta2-adrenoceptor gene polymorphisms. Respir Med. 2006;100:1072-78.

29. Bateman ED, Boushey HA, Bousquet J, et al. Can guideline-defined asthma control be achieved? The Gaining Optimal Asthma ControL study. Am J Respir Crit Care Med. 2004;170:836-44.

30. Pignatti PF. Trends in pharmacogenomics of drugs used in the treatment of asthma. Pharmacol Res. 2004:49:343-49.

31. Lima JJ, Thomason DB, Mohamed MH, Eberle LV, Self TH, Johnson JA. Impact of genetic polymorphisms of the beta2-adrenergic receptor on albuterol bronchodilator pharmacodynamics. Clin Pharmacol Ther. 1999;65:519-25.

32. Martinez FD, Graves PE, Baldini M, Solomon S, Erickson R. Association between genetic polymorphisms of the beta2-adrenoceptor and response to albuterol in children with and without a history of wheezing. J Clin Invest. 1997; 100:3184-88

33. Israel E, Chinchilli VM, Ford JG, et al. Use of regularly scheduled albuterol treatment in asthma: genotype-stratified, randomised, placebo-controlled crossover trial. Lancet. 2004:364:1505-12.

34. Taylor DR, Drazen JM, Herbison GP, Yandava CN, Hancox RJ, Town GI. Asthma exacerbations during long term beta agonist use: influence of beta(2) adrenoceptor polymorphism. Thorax. 2000;55:762-67.

35. Israel E, Drazen JM, Liggett SB, et al. The effect of polymorphisms of the beta(2)-adrenergic receptor on the response to regular use of albuterol in asthma. Am J Respir Crit Care Med. 2000;162:75-80.

36. Anderson W, Kalberg C, Edwards L, et al. Effects of polymorphisms in the promoter region of 5-lipooxygenase and LTC4 synthase on the clinical response to zafirlukast and fluticasone. Eur Resp J. 2000:16(suppl B):183S

37. Bray PJ, Cotton RG. Variations of the human glucocorticoid receptor gene (NR3C1): pathological and in vitro mutations and polymorphisms. Hum Mutat. 2003;21:557-68

38. Leung DY, Martin RJ, Szefler SJ, et al. Dysregulation of interleukin 4, interleukin 5, and interferon gamma gene expression in steroid-resistant asthma. J Exp Med. 1995;181:33-40.

39. Leung DY, Bloom JW. Update on glucocorticoid action and resistance. J Allergy Clin Immunol. 2003;111:3-22.

40. Umland SP, Schleimer RP, Johnston SL. Review of the molecular and cellular mechanisms of action of glucocorticoids for use in asthma. Pulm Pharmacol Ther. 2002;15:35-50.
41. Tantisira KG, Lake S, Silverman ES, et al. Corticosteroid pharmacogenetics: association of sequence variants in CRHRl with improved lung function in asthmatics treated with inhaled corticosteroids. Hum Mol Genet. 2004;13:135359 .

42. Obase Y, Shimoda T, Kawano T, et al. Polymorphisms in the CYP1A2 gene and theophylline metabolism in patients with asthma. Clin Pharmacol Ther. 2003; 73:468-74

43. Wechsler ME, Lehman E, Lazarus SC, et al. Beta-adrenergic receptor polymorphisms and response to salmeterol. Am J Respir Crit Care Med. 2006;173:519-26

44. Joos L, Sandford AJ. Genotype predictors of response to asthma medications. Curr Opin Pulm Med. 2002;8:9-15.

45. Renzi PM. Antileukotriene agents in asthma: the dart that kills the elephant? CMAJ. 1999;160:217-23.

46. Leff AR. Regulation of leukotrienes in the management of asthma: biology and clinical therapy. Annu Rev Med. 2001;52:1-14.

47. Sampson AP, Siddiqui S, Buchanan D, et al. Variant LTC(4) synthase allele modifies cysteinyl leukotriene synthesis in eosinophils and predicts clinical response to zafirlukast. Thorax. 2000;55(suppl 2):S28-S31.

48. Drazen JM, Yandava CN, Dubé L, et al. Pharmacogenetic association between ALOX5 promoter genotype and the response to anti-asthma treatment [letter]. Nat Genet. 1999;22:168-70.

49. Leung DY, Spahn JD, Szefler SJ. Steroid-unresponsive asthma. Semin Respir Crit Care Med. 2002;23:387-98.

50. Corrigan CJ, Brown PH, Barnes NC, et al. Glucocorticoid resistance in chronic asthma. Glucocorticoid pharmacokinetics, glucocorticoid receptor characteristics, and inhibition of peripheral blood T cell proliferation by glucocorticoids in vitro. Am Rev Respir Dis. 1991;144:1016-25.

51. Ito K, Chung KF, Adcock IM. Update on glucocorticoid action and resistance. J Allergy Clin Immunol. 2006;117:522-43.

52. Chan MT, Leung DY, Szefler SJ, Spahn JD. Difficult-to-control asthma: clinical characteristics of steroid-insensitive asthma. J Allergy Clin Immunol. 1998;101:594-601.

53. Raby BA, Van Steen K, Lazarus R, Celedon JC, Silverman EK, Weiss ST. Eotaxin polymorphisms and serum total IgE levels in children with asthma. J Allergy Clin Immunol. 2006;117:298-305.

54. Davydov L. Omalizumab (Xolair) for treatment of asthma. Am Fam Physician. 2005; 71:341-42.

55. Veenstra DL, Higashi MK, Phillips KA. Assessing the cost-effectiveness of pharmacogenomics. AAPS PharmSci. 2000;2:E29.

56. Duffy MJ. Predictive markers in breast and other cancers: a review. Clin Chem. 2005;51:494-503.

57. Smith I, Procter M, Gelber RD, et al. 2-year follow-up of trastuzumab after adjuvant chemotherapy in HER2-positive breast cancer: a randomised controlled trial. Lancet. 2007;369:29-36.

58. Lee JS, Thorgeirsson SS. Genetic profiling of human hepatocellular carcinoma. Semin Liver Dis. 2005;25:125-32.

59. Joensuu H, Roberts PJ, Sarlomo-Rikala M, et al. Effect of the tyrosine kinase inhibitor STI571 in a patient with a metastatic gastrointestinal stromal tumor. N Engl J Med. 2001;344:1052-56.

60. Walsh MJ, Jonsson JR, Richardson MM, et al. Non-response to antiviral therapy is associated with obesity and increased hepatic expression of suppressor of cytokine signalling 3 (SOCS-3) in patients with chronic hepatitis C, viral genotype 1. Gut. 2006;55:529-35.

61. Lerer B, Macciardi F. Pharmacogenetics of antidepressant and mood-stabilizing drugs: a review of candidate-gene studies and future research directions. Int J Neuropsychopharmacol. 2002;5:255-75. 
62. Reynolds GP, Yao Z, Zhang X, Sun J, Zhang Z. Pharmacogenetics of treatment in first-episode schizophrenia: D3 and 5-HT2C receptor polymorphisms separately associate with positive and negative symptom response. Eur Neuropsychopharmacol. 2005;15:143-51.

63. Israel E. Introduction: the rise of the age of individualism-variability in the pathobiology, response to treatment, and treatment outcomes in asthma. J Allergy Clin Immunol. 2005;115:S525.

64. Israel E. Genetics and the variability of treatment response in asthma J Allergy Clin Immunol. 2005;115:S532-S538.
65. Weiss ST, Lake SL, Silverman ES, et al. Asthma steroid pharmacogenetics: a study strategy to identify replicated treatment responses. Proc Am Thorac Soc. 2004; 1:364-67.

66. Stallings SC, Huse D, Finkelstein SN, et al. A framework to evaluate the economic impact of pharmacogenomics. Pharmacogenomics. 2006;7:853-62.

67. Weiss ST, Litonjua AA, Lange C, et al. Overview of the pharmacogenetics of asthma treatment. Pharmacogenomics J. 2006;6:311-26. 